\title{
Morfometría del burro criollo de la región de Zozutla, Puebla, México
}

\section{Morphometry of creole donkey in the Zozutla region, Puebla, Mexico}

Reséndiz Martínez Roberto ${ }^{1}$, Romero Castañón Salvador ${ }^{1 凶}$, Juárez Cortez Juan A. ${ }^{1}$, Jiménez Cortez Herminio I. ${ }^{1}$, Covarrubias Balderas Armando ${ }^{1}$, Lázaro Galicia Cecilia ${ }^{1}$ y Becerra Peralta Fernando ${ }^{1}$

${ }^{1}$ Facultad de Medicina Veterinaria y Zootecnia. Benemérita Universidad Autónoma de Puebla.

هutor para correspondencia: $\underline{\text { salvador.romero@correo.buap.mx }}$

Recibido: $26 / 09 / 2019$

Aceptado: 19/11/2019

\section{RESUMEN}

En México el burro es una especie con importancia económica en las zonas rurales, ya que se utiliza como medio de transporte y carga, siendo una ayuda indispensable al campesino marginado, que vive en regiones remotas y mal comunicadas. El objetivo de la presente investigación fue el de caracterizar la morfometría del burro criollo de la región de Zozutla, Puebla. México. Se estudiaron 25 asnos hembras y 25 asnos machos, de 2 a 3 años. A cada uno de los animales de estudio, aplomados correctamente y accediendo a los mismos por su flanco izquierdo, se les tomaron sus medidas corporales o variables zoométricas de naturaleza cuantitativa, por medio de la cinta métrica y el pelvímetro: alzada de la cruz (AC), alzada dorso (AD), alzada grupa (AG), alzada pelvis (AP), alzada palomillas (APa), alzada nacimiento cola (ANC), diámetro longitudinal (DL), diámetro dorso esternal (DDE), diámetro entre encuentros (DEE), diámetro bicostal (DB), anchura grupa $(\mathrm{AnG})$, longitud grupa (LG), perímetro torácico $(\mathrm{PT})$, perímetro rodilla (PR), perímetro caña (PC), perímetro menudillo $(\mathrm{PM})$, perímetro cuartilla $(\mathrm{PCu})$, perímetro corona $(\mathrm{PCo})$,perímetro corvejón (PCor), longitud oreja (LO), longitud cabeza (LC), anchura cabeza (ACa), profundidad cabeza (PCa), longitud cara ( $\mathrm{LCa})$ y anchura cráneo ( $\mathrm{ACr}$ ). Los resultados obtenidos a partir de las 26 variables de medición biométricas proporcionaron los datos importantes para diferenciar los burros criollos hembras de los machos, y fueron agrupados en conjuntos específicos en base a las variables de estudio, proporcionando en los animales las aptitudes funcionales. Los resultados demostraron que las variables muestran diferencias altamente significativas $(\mathrm{P}<.0001)$ en las mediciones de los animales de estudio. En esta investigación los animales criollos, fueron de talla pequeña, los cuales presentaron una deficiente alimentación y un nulo manejo sanitario de los animales.

Palabras clave: Burros, variables zoométricas, mediciones.

\begin{abstract}
In Mexico, the donkey is a species with economic importance in rural areas as it is used as a means of transport and cargo, being an indispensable aid to the marginalized farmer, who lives in remote and poorly communicated regions. The objective of this research was to characterize the morphometry of the Creole donkey of the Zozutla region, Puebla. Mexico. 25 female asses and 25 male asses, from 2 to 3 years old, were studied. Each of the study animals, correctly plumbed and
\end{abstract}


accessed by their left flank, were taken their body measurements or zoometric variables of quantitative nature, by means of the measuring tape and the pelvimeter; cross elevation (AC), back elevation (AD), rump elevation (AG), pelvis elevation (AP), palomillas elevation (APa), tail birth elevation (ANC), longitudinal diameter (DL), sternal back diameter (DDE), diameter between encounters (DEE), bicostal diameter (DB), rump width (AnG), rump length (LG), thoracic perimeter (PT), knee perimeter (PR), cane perimeter (PC), girth perimeter (PM), quarter perimeter $(\mathrm{PCu})$, crown perimeter (PCo), hock perimeter (PCor), ear length (LO), head length (LC), head width (ACa), head depth (PCa), face length (LCa) and width skull (ACr). The results obtained from the 26 biometric measurement variables provided the important data to differentiate female creole donkeys from males, were grouped into specific sets based on the study variables, providing functional aptitudes in animals. The results showed that the variables show highly significant differences $(\mathrm{P}<.0001)$ in the measurements of the study animals. In this investigation the Creole animals were small in size, which presented poor feeding and no sanitary management of the animals.

Keywords: Donkeys, zooms variables, measurements.

\section{INTRODUCCIÓN}

En México los equinos (caballos, mulas y burros) siguen siendo una ayuda indispensable al campesino marginado, que vive en regiones remotas y mal comunicadas. Sin embargo, poco se ha hecho para mejorarlas. Al contrario, a través de siglos de mala alimentación y los abusos en todos los sentidos, los animales han adquirido defectos de conformación, especialmente de sus extremidades; su tamaño menor y sus huesos más delgados comparados con sus ancestros europeos de hoy. Se estima que en México existen 3,270,000 burros (Shunemann, et al, 2001) dedicados a diferentes labores, distribuidos en toda la República Mexicana, habitando principalmente en áreas rurales y urbanas en donde existen comunidades en extrema pobreza que necesitan de sus servicios.

En 1995, la Organización de las Naciones Unidas para la Agricultura y la Alimentación, calculó que de los 4000 a 5000 recursos genéticos animales existentes en el mundo, de 1200 a 1500 se encontraban en peligro de desaparición (FAO, http://fao.org). Estimó que el 30\% de las razas domésticas de ganado corrían peligro de extinción, desapareciendo cada mes unas 6 razas en el mundo y que más de la mitad de éstos, se perdían en países desarrollados (F.A.O., 1995).

\section{MATERIALES Y MÉTODOS}

El estudio morfoestructural se realizó sobre una muestra de 50 animales, 25 asnos hembras y 25 asnos machos, de 3 años de edad, de la región de Zozutla, Puebla (figura 1). Se localiza en el Municipio Yehualtepec del Estado de Puebla México y se encuentra en las coordenadas GPS: Longitud (dec): 97.681944, Latitud (dec): 18.758889. La localidad se encuentra a una mediana altura de 2000 metros sobre el nivel del mar. A cada uno de los animales muestreados, aplomados correctamente y accediendo a los mismos por su flanco izquierdo, se les tomaron 26 medidas corporales (tabla 1) o variables zoométricas de naturaleza cuantitativa, las cuales se dividieron en tres grupos de acuerdo a sus regiones corporales: medidas cefálicas, troncales y de extremidades. Con el apoyo de 
la cinta métrica, el pelvímetro y del bastón zoométrico se procedió a medir la alzada de la cruz (AC), alzada dorso (AD), alzada grupa (AG), alzada pelvis (AP), alzada palomillas (APa), alzada nacimiento cola (ANC), diámetro longitudinal (DL), diámetro dorso esternal (DDE), diámetro entre encuentros (DEE), diámetro bicostal (DB), anchura grupa (AnG), longitud grupa (LG), perímetro torácico $(\mathrm{PT})$, perímetro rodilla $(\mathrm{PR})$, perímetro caña (PC), perímetro menudillo $(\mathrm{PM})$, perímetro cuartilla $(\mathrm{PCu})$, perímetro corona (PCo),perímetro corvejón (PCor), longitud oreja (LO), longitud cabeza (LC), anchura cabeza (ACa), profundidad cabeza (PCa), longitud cara (LCa) y anchura cráneo (ACr). El análisis de los datos se realizará mediante un análisis de Varianza (ANOVA), el cual se elaboró en el programa estadístico SAS versión 9.4.

\section{RESULTADOS Y DISCUSIÓN}

La comparación de los resultados obtenidos (graficas 2,3,4,5,6,7,8, y 9) con los de otros autores, revela diferencias, las cuales pudiesen ser debido a un mínimo margen de error en las mediciones, siendo esto inevitable en muchas de las mediciones, ya que la localización exacta de los puntos anatómicos en la toma de las medidas zoométricas, no fue tan sencilla, por lo que es relativamente fácil que diferentes personas, obtengan mediciones ligeramente distintas, en un mismo animal. López (1993), Soltillo y Serrano (1985), obtuvieron valores medios en las medidas de alzada de la cruz de $150 \mathrm{~cm}$, mientras que García (2006), obtuvo $137 \mathrm{~cm}$, mientras que la medición de la alzada de la cruz en la presente investigación fue de $103 \mathrm{~cm}$. Esta diferencia se da por el hecho, de que en esta investigación se utilizaron animales criollos, de talla pequeña, con una deficiente alimentación y un nulo manejo sanitario de los mismos.

Tabla 1. Simbología de Variables de Estudio

\begin{tabular}{|r|c|}
\hline 1 & Alzada a la cruz \\
\hline 2 & Alzada al dorso \\
\hline 3 & Alzada a al entrada de la grupa \\
\hline 4 & Alzada a la pelvis \\
\hline 5 & Alzada a las palomillas \\
\hline 6 & Alzada nacimiento cola \\
\hline 7 & Diametro longitudinal \\
\hline 8 & Diametro dorso - esternal \\
\hline 9 & Dimetro entre encuentros \\
\hline 10 & Diametro bicostal \\
\hline 11 & Anchura grupa \\
\hline 12 & Longitud grupa \\
\hline 13 & Longitud cabeza \\
\hline 14 & Profundidad cabeza \\
\hline 15 & Anchura cabeza \\
\hline 16 & Longitud cara \\
\hline 17 & Longitud craneo \\
\hline 18 & Anchura craneo \\
\hline 19 & Perimetro toracico \\
\hline 20 & Perimetro rodilla \\
\hline 21 & Perimetro caña \\
\hline 22 & Perimetro menudillo \\
\hline 23 & Perimetro cuartilla \\
\hline 24 & Perimetro corona \\
\hline 25 & Periemtro corvejon \\
\hline 26 & Longitud oreja \\
\hline & \\
\hline
\end{tabular}


El análisis de los datos se realizó mediante un análisis de Varianza (ANOVA), el cual se elaboró en el programa estadístico SAS versión 9.4. Los resultados obtenidos proporcionaron datos importantes para diferenciar entre burros hembras y machos, para agruparlos en conjuntos específicos $\mathrm{y}$, fundamentalmente, para deducir proporciones que a su vez indiquen aptitudes funcionales.
Los resultados que nos arrojó el estudio fue que las variables muestran diferencias altamente significativas $(\mathrm{P}<.0001)$ en las mediciones de los animales de estudio.

A continuación, se presenta una prueba de medias de Tukey para todas las variables, la cual nos indica si hembras o machos presentan mayor valor morfométrico.

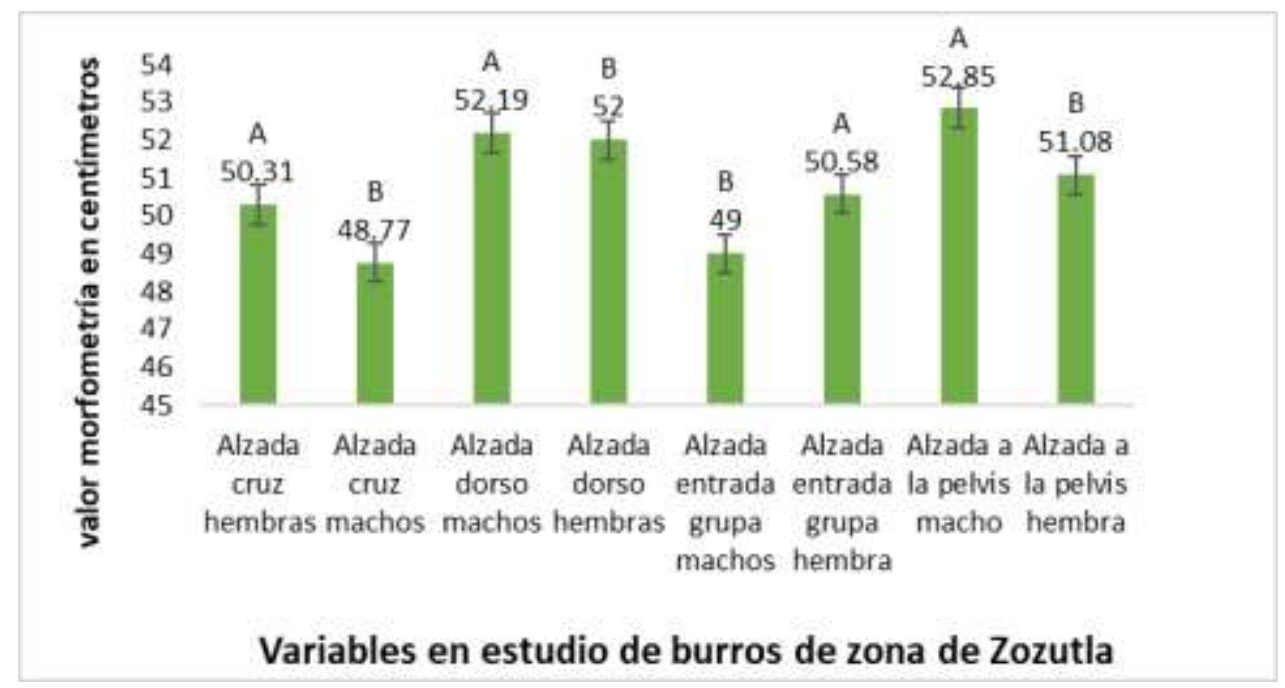

Gráfica 2. Prueba de medias de Tukey para estudio morfométrico en las variables: Alzada de cruz en hembras y machos, alzada de dorso hembras y machos, alzada entrada grupa hembras y machos, alzada de pelvis hembras y machos, (letra A y B nos indican la diferencia estadística entre cada sexo)

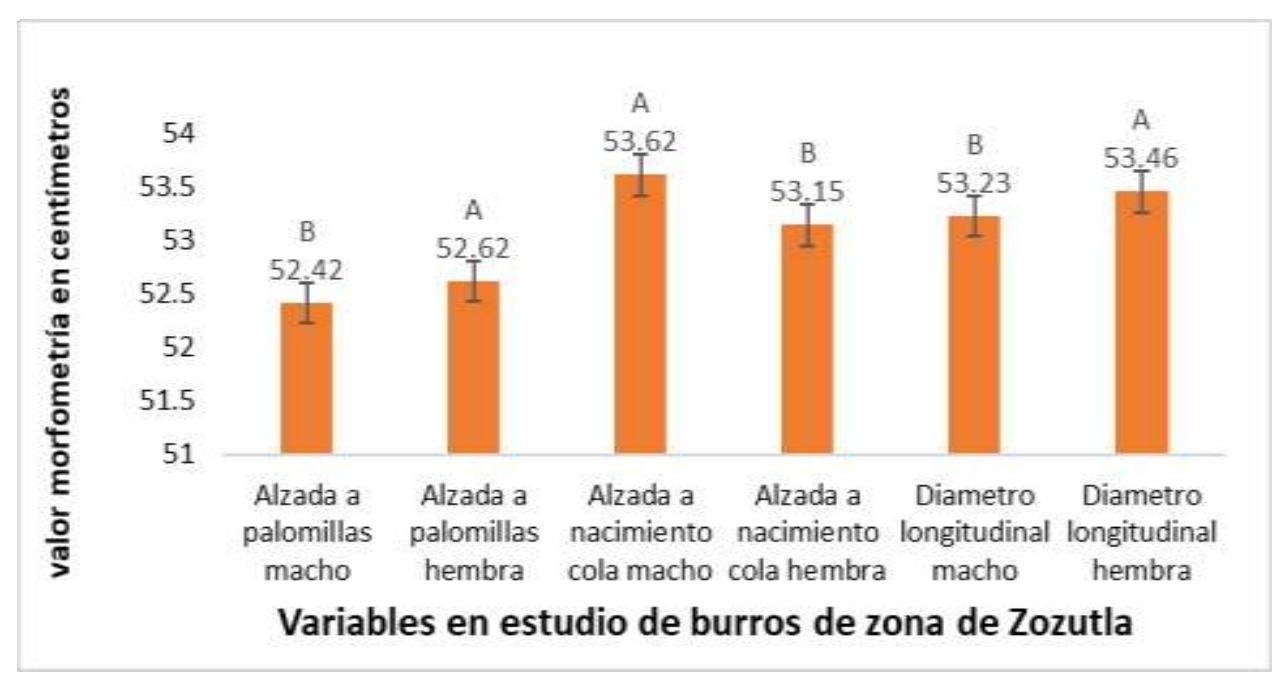

Gráfica 3. Prueba de medias de Tukey para estudio morfométrico en las variables: Alzada de palomillas en hembras y machos, alzada de nacimiento cola hembras y machos, diámetro longitudinal entre hembra y macho, (letra A y B nos indican la diferencia estadística entre cada sexo). 
Reséndiz et al., 2019

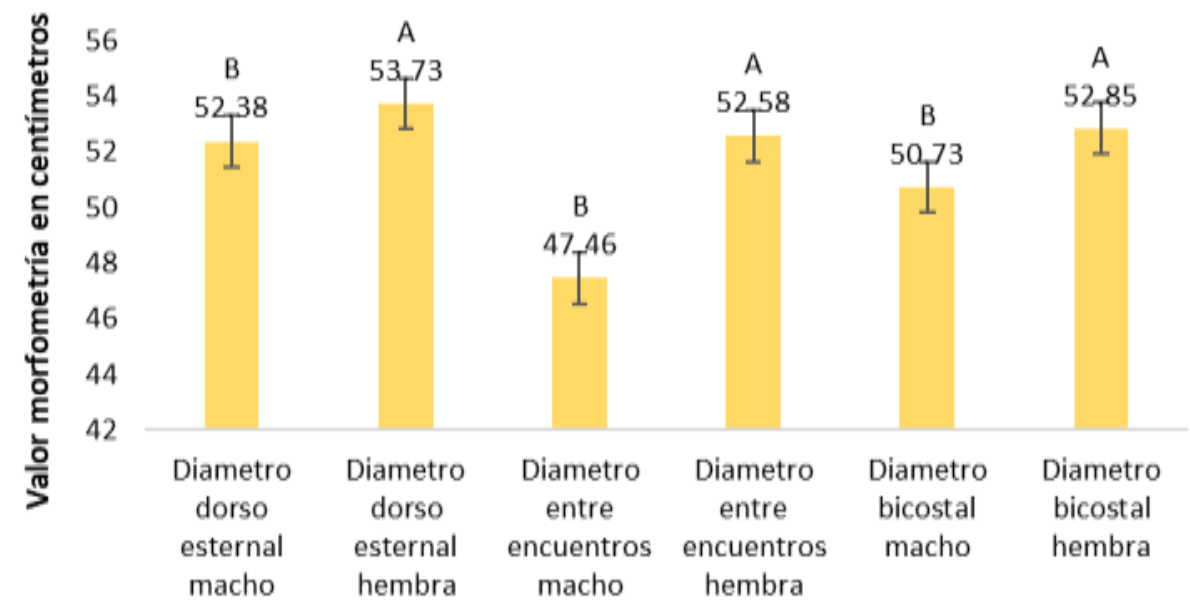

Variables en estudio de burros de zona de Zozutla

Gráfica 4. Prueba de medias de Tukey para estudio morfométrico en las variables: Diámetro de dorso esternal entre hembras y machos, diámetro entre encuentros hembras y machos, diámetro bicostal entre hembra y macho, (letra A y B nos indican la diferencia estadística entre cada sexo).

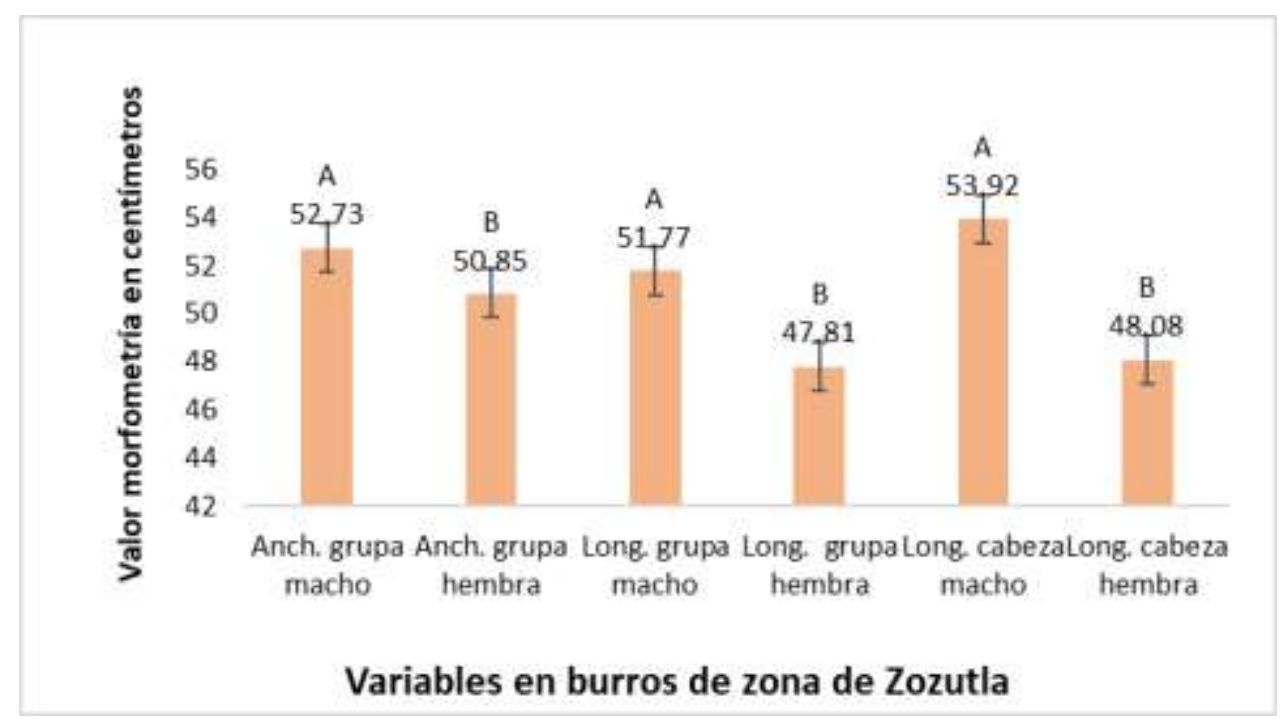

Gráfica 5. Prueba de medias de Tukey para estudio morfométrico en las variables: Ancho de grupa entre hembras y machos, longitud de grupa entre hembras y machos, longitud de cabeza entre hembra y macho, (letra $\mathrm{A}$ y $\mathrm{B}$ nos indican la diferencia estadística entre cada sexo). 


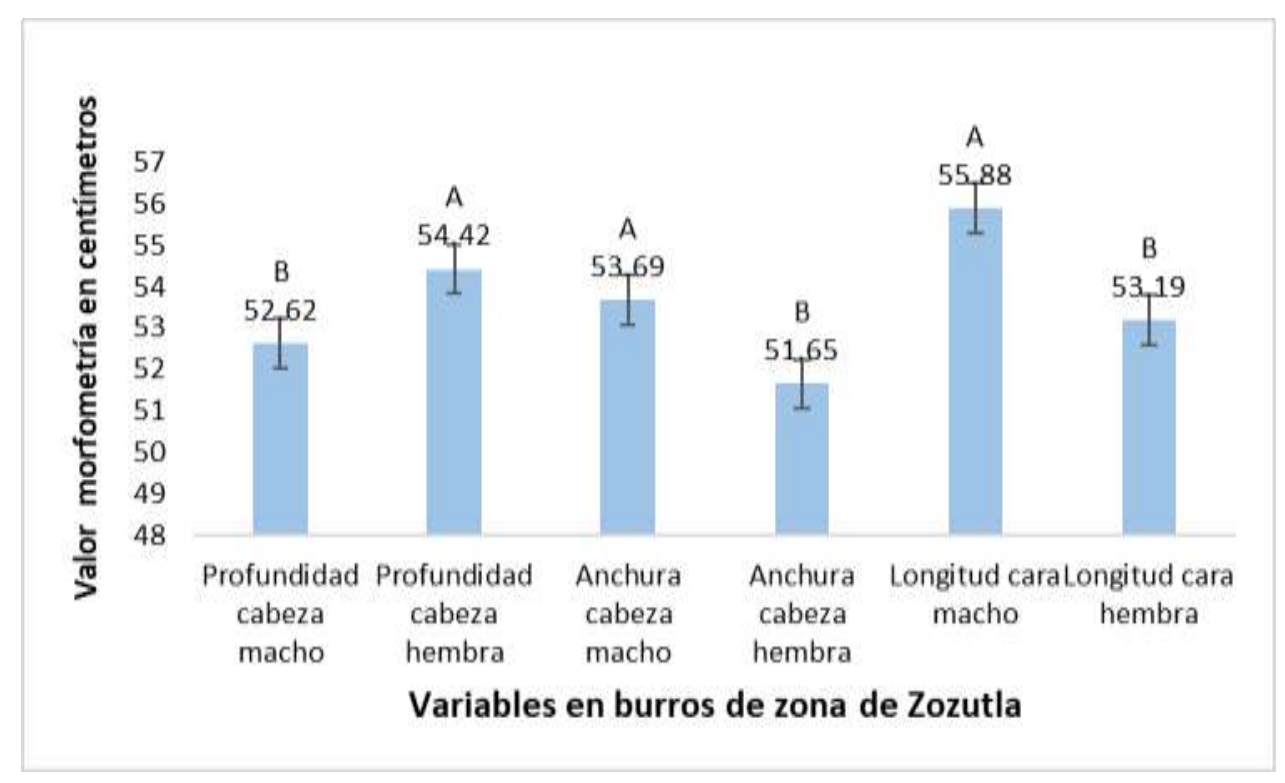

Gráfica 6. Prueba de medias de Tukey para estudio morfométrico en las variables: Profundidad de cabeza entre hembras y machos, Anchura de cabeza entre hembras y machos, longitud de cara entre hembra y macho, (letra A y B nos indican la diferencia estadística entre cada sexo).

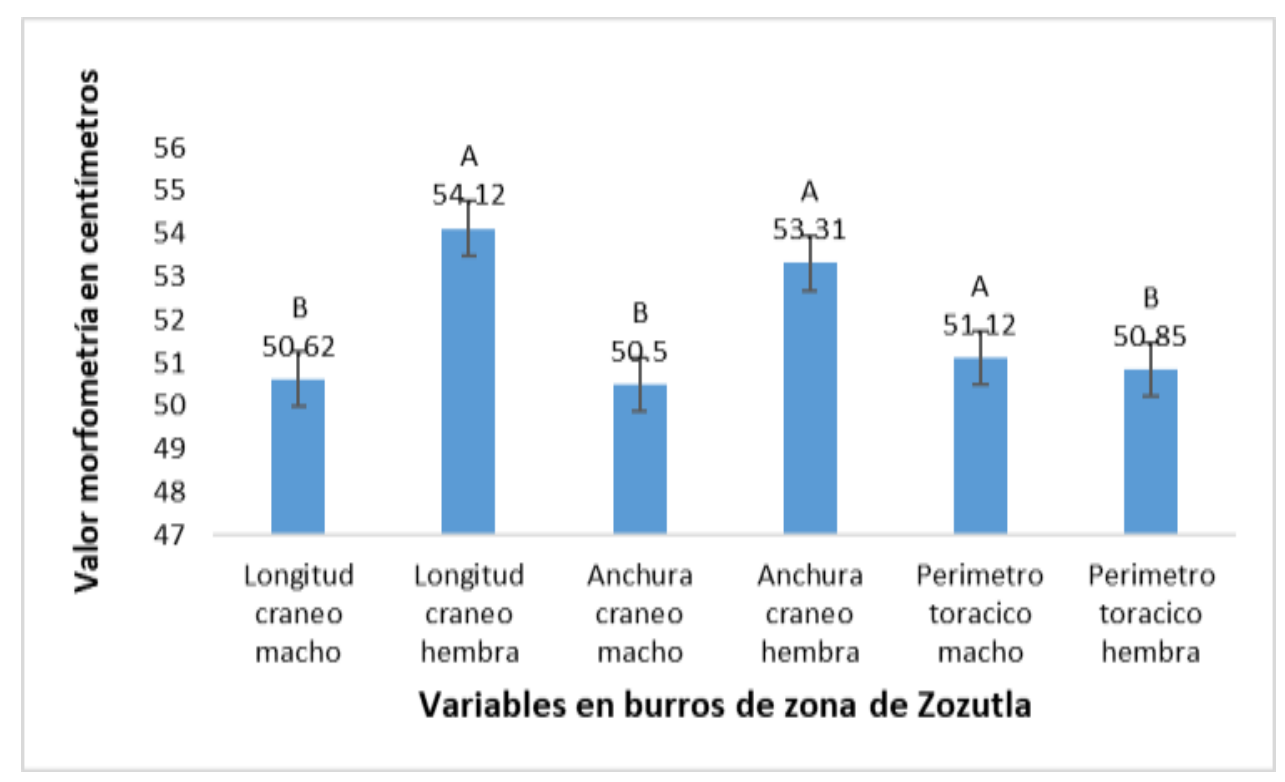

Gráfica 7. Prueba de medias de Tukey para estudio morfométrico en las variables: Longitud de cráneo entre hembras y machos, Anchura de cráneo entre hembras y machos, Perímetro torácico entre hembra y macho, (letra A y B nos indican la diferencia estadística entre cada sexo). 


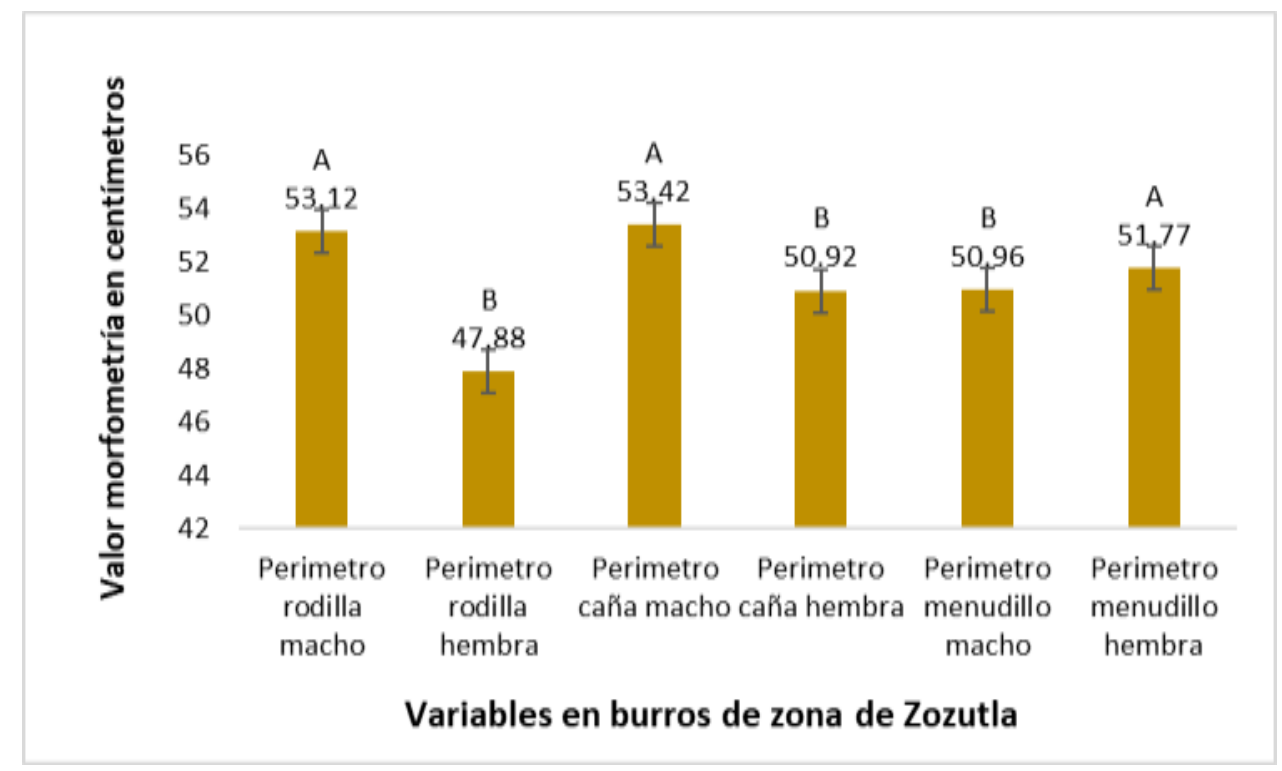

Gráfica 8. Prueba de medias de Tukey para estudio morfométrico en las variables: Perímetro de rodilla entre hembras y machos, perímetro de caña entre hembras y machos, Perímetro de menudillo entre hembra y macho, (letra A y B nos indican la diferencia estadística entre cada sexo).

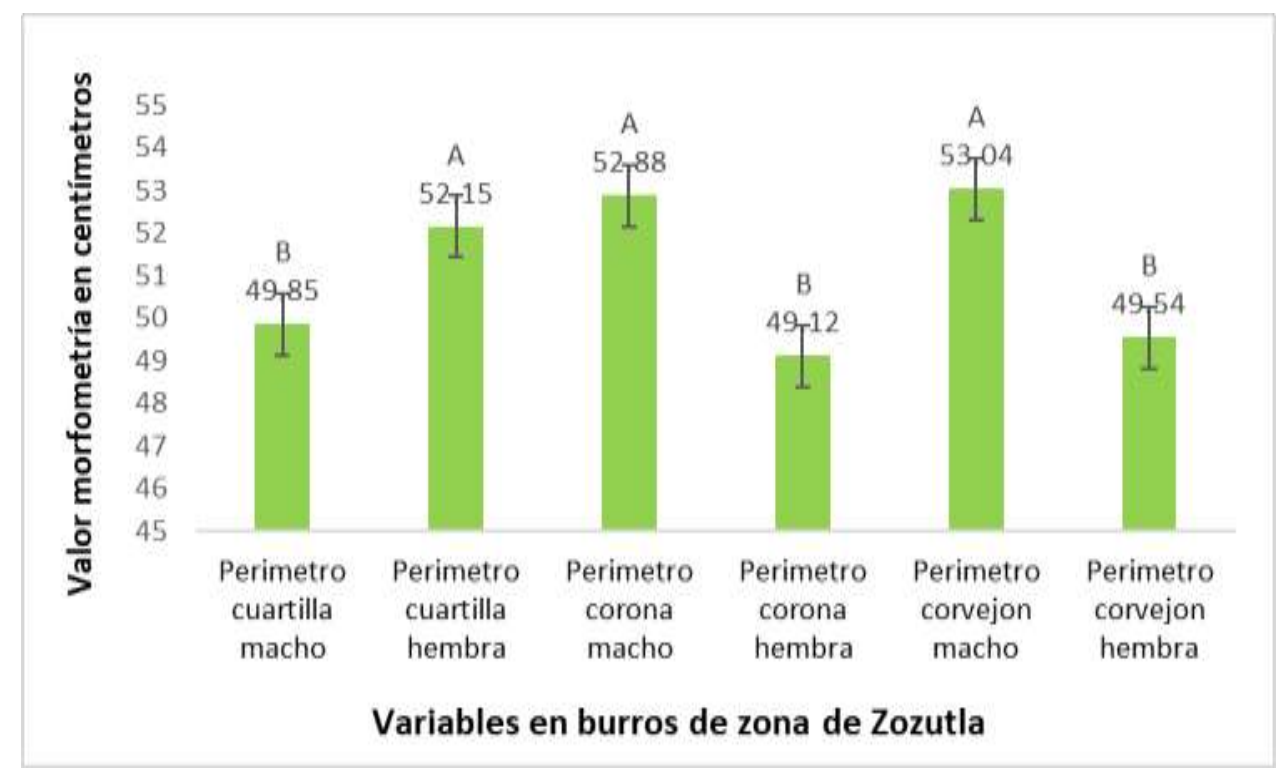

Gráfica 9. Prueba de medias de Tukey para estudio morfométrico en las variables: Perímetro de cuartilla entre hembras y machos, perímetro de corona entre hembras y machos, Perímetro de corvejón entre hembra y macho, (letra A y B nos indican la diferencia estadística entre cada sexo). 


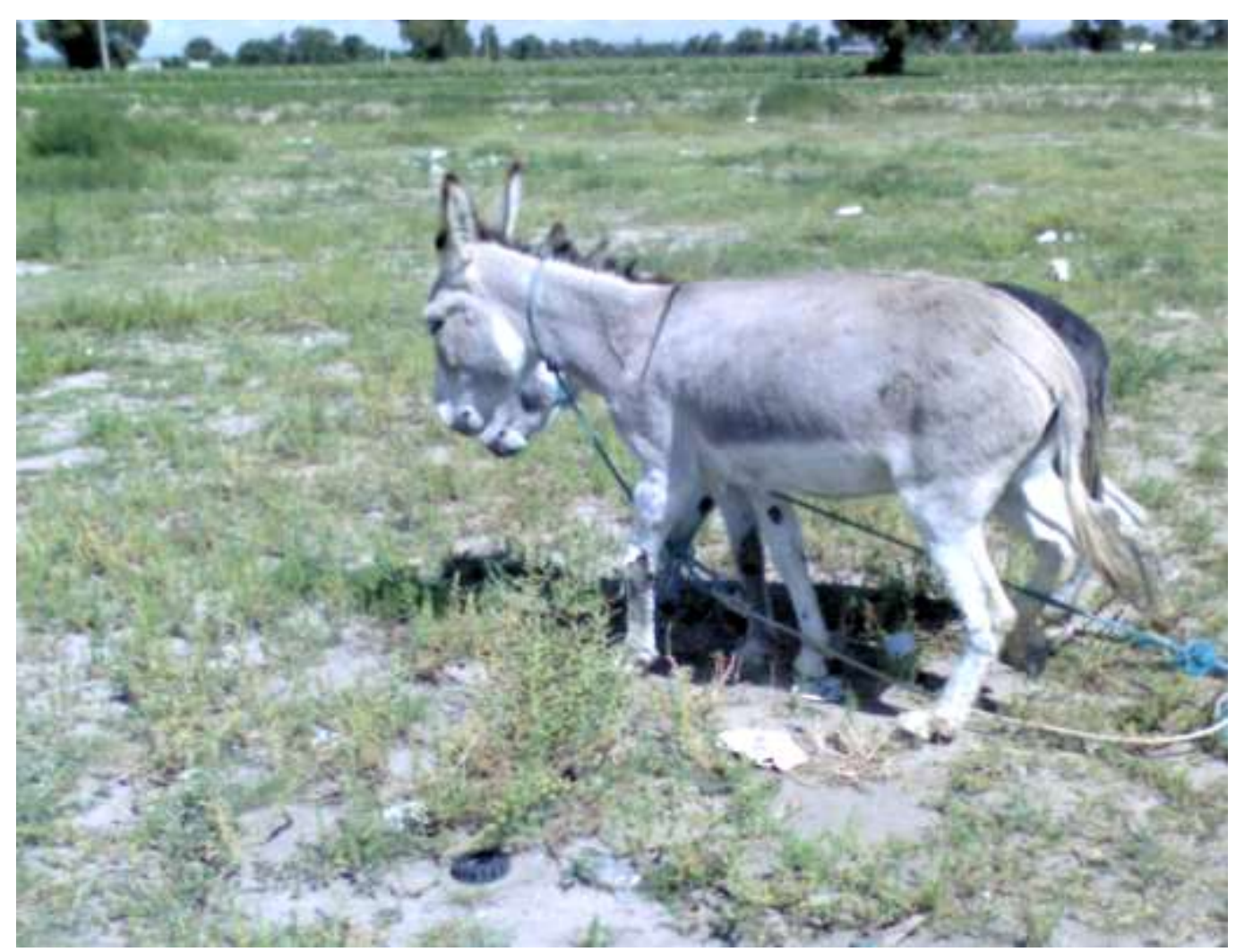

Figura 1. Burros de estudio de la región de Zozutla, Puebla.

\section{CONCLUSION}

El estudio evidencia que el burro Criollo de Zozutla, que se traduce en una constitución no tan proporcionada, equilibrada, armónica y con una buena aptitud para el trabajo; se aprecia un marcado dimorfismo sexual en favor de los machos, típico en los équidos. Se concluyó que hasta los 2 años de edad los animales tuvieron un crecimiento alométrico donde los machos alcanzaron medidas superiores a las hembras, luego de lo cual el crecimiento fue mínimo en las hembras en determinadas medidas incluso superaron a los machos.

\section{LITERATURA CITADA}

FAO. 1995. World watch list for Domestic Animal Diversity. $2^{\text {nd }}$ Edition. FAO, Rome.
García., M.E. (2006). Caracterización morfológica, hematológica y bioquímica clínica en 5 razas asnales españolas para programas de conservación. Tesis de Doctorado en Veterinaria. Universidad Autónoma de Barcelona. Bellaterra, España.

López, J.M. (1993). Razas asnales de España. XV Curso de especialización de cría caballar.

Shunemann de AA, Bouda J, López CA, Chavira SH. Valores Bioquímicos en sangre de burros antes y después del trabajo. Vet Méx 2001; 32(4):271-278.

Soltillo, J.L., Serrano, V. (1985). Producción animal. I Etnología Zootécnica. TebarFlores (ed) Madrid. 
Copyright (c) 2019 Roberto Reséndiz Martinez, Salvador Rom ero Castañón, Juan A. Juárez Cortez, Herm inio I. Jiménez Cortez, Armando Covarrubias Balderas, Cecilia Lázaro Galicia y Fernando Becerra Peralta

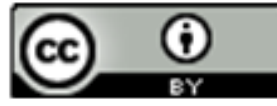

Este texto está protegido por una licencia licencia CreativeCommons 4.0.

Usted es libre para Compartir — copiar y redistribuir el material en cualquier medio o formato-y Adaptar el documento —remezclar, transformar y crear a partir del material- para cualquier propósito, incluso para fines com erciales, siempre que cumpla la condición de:

Atribución: Usted debe dar crédito a la obra original de manera adecuada, proporcionar un enlace a la licencia, e indicar si se han realizado cambios. Puede hacerlo en cualquier forma razonable, pero no de forma tal que sugiera que tiene el apoyo del licenciante o lo recibe por el uso que hace de la obra.

Resumendelicencia - Textocompletodelalicencia 